\title{
FRACTIONAL DIFFERENTIAL OPERATORS IN VECTOR-VALUED SPACES AND APPLICATIONS
}

\author{
VELI SHAKHMUROV
}

Abstract. Fractional differential operator equations with parameter are studied. Uniform $L_{p}$ separability properties and sharp resolvent estimates are obtained for elliptic equations in terms of fractional derivatives. Moreover, maximal regularity properties of the fractional abstract parabolic equation are established. Particularly, it is proven that the operators generated by these equations are positive and also are generators of analytic semigroups. As an application, the anisotropic parameter dependent fractional differential equations and the system of fractional differential equations are studied.

Mathematics subject classification (2010): 35R11, 35J25, 35K90, 47F05, 43A95.

Keywords and phrases: Fractional-differential equations, Sobolev-Lions spaces, differential-operator equations, maximal $L_{p}$ regularity, abstract parabolic equations, operator-valued multipliers.

\section{REFERENCES}

[1] Amann H., Linear and quasi-linear equations, 1, Birkhauser, Basel 1995.

[2] Agarwal R., Bohner M., Shakhmurov V. B., Maximal regular boundary value problems in Banach-valued weighted spaces, Bound. Value Probl., (1)(2005), 9-42.

[3] Ashyralyev A., Sharifov Y. A., Existence and uniqueness of solutions for the system of nonlinear fractional differential equations with nonlocal and integral boundary conditions, Abstr. Appl. Anal., ID 594802, (2012).

[4] BURKHOLDER D. L., A geometrical conditions that implies the existence certain singular integral of Banach space-valued functions, Proc. conf. Harmonic analysis in honor of Antonu Zigmund, Chicago, 1981,Wads Worth, Belmont, (1983), 270-286.

[5] Dore C. AND Yakubov S., Semigroup estimates and non coercive boundary value problems, Semigroup Forum 60 (2000), 93-121.

[6] DubinskiI Yu. A., The Cauchy problem and pseudodifferential operators in the complex domain, Uspekhi Mat. Nauk, 45:2(272) (1990), 115-142.

[7] Diestel J., Jarchow H., Tonge A., Absolutely summing operators, Cambridge Univ. Press, Cambridge, 1995.

[8] Denk R., Krainer T., R-Boundedness, pseudodifferential operators, and maximal regularity for some classes of partial differential operators, Manuscripta Math. 124(3) (2007), 319-342.

[9] Haller R., Heck H., Noll A., Mikhlin's theorem for operator-valued Fourier multipliers in $n$ variables, Math. Nachr. 244 (2002), 110-130.

[10] Hermander L., The analysis of linear partial differential operators, V. 3, 4, Springer-Verlag, New York, 1985.

[11] FAVINi A., ShaKhmURov V. B., YAKOV YAKUBOV, Regular boundary value problems for complete second order elliptic differential-operator equations in UMD Banach spaces, Semigroup form, 79 (1)(2009), 22-54.

[12] Krein S. G., Linear differential equations in Banach space, American Mathematical Society, Providence, 1971.

[13] Lizor Kin P. I., Generalized Liouville differentiation and functional spaces $L_{p}^{r}\left(E_{n}\right)$, Embedding theorems, Mathematics of the USSR-Sbornik (3)60 (1963), 325-353. 
[14] NAGASE M., The $L_{p}$-boundedness of pseudodifferential operators with non-regular symbols, Comm. in Partial Differential Equations, 2 (10), (1977), 1045-1061.

[15] Portal P., ŠTrkalu Ž., Pseudodifferential operators on Bochner spaces and an application, Math. Z. 253 (2006), 805-819.

[16] Shakhmurov V. B., Coercive boundary value problems for regular degenerate differential-operator equations, J. Math. Anal. Appl., 292 (2) (2004), 605-620.

[17] Shakhmurov V. B., Embedding and separable differential operators in Sobolev-Lions type spaces, Mathematical Notes, (84) 6, (2008), 906-926.

[18] Shakhmurov V. B., Maximal regular abstract elliptic equations and applications, Siberian Math. Journal, (51) 5, (2010), 935-948.

[19] Shakhmurov V. B., Embedding and maximal regular differential operators in Banach-valued weighted spaces, Acta Math. Sinica, 22(5) (2006), 1493-1508.

[20] TRIEBEL H., Interpolation theory, Function spaces, Differential operators, North-Holland, Amsterdam, 1978.

[21] TAYlor M., Pseudo-differential operators, Princeton University press, Princeton, NJ, 1981.

[22] Treves F., Introduction to pseudo-differential and Fourier integral operators, Plenum press, New York and London, 1980.

[23] WeIs L., Operator-valued Fourier multiplier theorems and maximal $L_{p}$ regularity, Math. Ann. 319(2001), 735-758.

[24] Yakubov S., Yakubov Ya., Differential-operator Equations. Ordinary and Partial Differential Equations, Chapman and Hall /CRC, Boca Raton, 2000. 